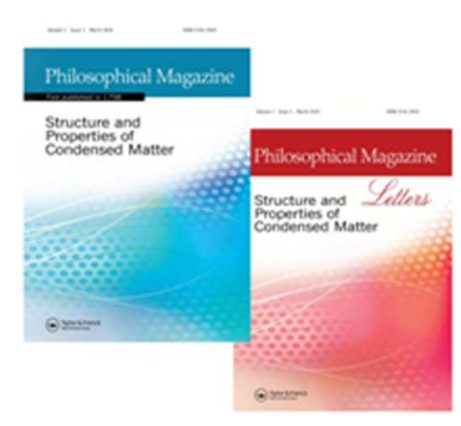

\title{
Statistics and Topological Changes in 2D Foam from the Dry to the Wet Limit
}

\begin{tabular}{|r|l|}
\hline Journal: & Philosophical Magazine \& Philosophical Magazine Letters \\
\hline Manuscript ID & TPHM-16-Aug-0368.R2 \\
\hline Dournal Selection: & Philosophical Magazine \\
\hline Complete List of Authors: & $\begin{array}{l}\text { Dunne, Friedrich; University of Dublin Trinity College, School of Physics } \\
\text { Bolton, Fintan; Red Hat GmbH, Werner-von-Siemens-Ring 14 } \\
\text { Weaire, Denis; Trinity College Dublin, Physics } \\
\text { Hutzler, Stefan; Trinity College Dublin, Physics }\end{array}$ \\
\hline Keywords: & foams, bubbles, numerical simulation \\
\hline Keywords (user supplied): & bubble side distribution, Foam excess energy, 2D wet foams \\
\hline & \multicolumn{2}{|l}{} \\
\hline
\end{tabular}




\title{
Statistics and Topological Changes in 2D Foam from the Dry to the Wet Limit
}

\author{
F.F. Dunne ${ }^{\mathrm{a}}$, F. Bolton ${ }^{\mathrm{b}}$, D. Weaire ${ }^{\mathrm{a}}$, and S. Hutzler ${ }^{\mathrm{a}}$ \\ ${ }^{a}$ School of Physics, Trinity College Dublin, The University of Dublin, Ireland; ${ }^{b}$ Red Hat \\ GmbH, Werner-von-Siemens-Ring 14, Technopark II, Haus C, Grasbrunn 85630, Germany
}

\author{
ARTICLE HISTORY \\ Compiled February 16, 2017
}

\begin{abstract}
The software PLAT, for the simulation of two-dimensional foams, has never been fully exploited, particularly in the wet limit, where it has difficulty converging. Present computer resources have enabled us to circumvent this problem. Using a dataset of 600000 simulations, we are able to compute the polydisperse foam excess energy all the way from the dry to the wet limit. We also present data and a simple empirical function for the number distribution of $n$-sided bubbles for all values of the average contact number $Z$. Furthermore we show that, starting from a dry foam, incremental increases in liquid fraction bring with them a steadily increasing number $n$ of bubbles involved in rearrangements. The size distribution of these rearrangements is found to be exponential, $p(n) \sim \exp (-\lambda n)$, where the mean, $1 / \lambda$, increases in the wet limit.
\end{abstract}

\section{KEYWORDS}

Foam excess energy; 2D foams; Simulation; Wet foams; Bubble side distribution

\section{Acknowledgements}

This publication has emanated from research supported in part by a research grant from Science Foundation Ireland (SFI) under [grant number 13/IA/1926]. This article is based upon work from COST Actions MP1106 'Smart and green interfaces' and MP1305 'Flowing matter', supported by COST (European Cooperation in Science and Technology). We also acknowledge the support of the European Space Agency ESA, Project microG-Foam, AO-99-075 and contract 4000115113, 'Soft Matter Dynamics'. Additional calculations were performed on the Lonsdale cluster maintained by the Trinity Centre for High Performance Computing. This cluster was funded through grants from Science Foundation Ireland (SFI).

\section{Introduction}

In the late $20^{\text {th }}$ century, considerable progress was made in the theory of liquid foams, but it was largely confined to relatively $d r y$ foams, i.e. foams with low liquid volume fraction $\phi$ [1]. For these we can benefit from both straightforward models and reliable 
numerical methods. More recently, attention has moved to the case of wet foams [2, 3], which pose new challenges.

In the wet limit, the shear modulus goes to zero (smoothly in the case of disordered foams with polydisperse bubble sizes), as was first confirmed from experiments by Princen and Kiss [4] and tentatively identified in the early two-dimensional (2D) PLAT simulation of Bolton and Weaire $[5,6,7,8]$ as well as in the 2D Bubble Model of Durian [9]. Also, the yield stress goes to zero. Again, Princen may be credited with this finding [10]. This trend was also reproduced in the early simulations of Bolton and Weaire [8] and Hutzler et al. [11]. An associated phenomenon was noted, which may be considered to be a third aspect of the critical behaviour associated with the wet limit: it might be termed fragility. This is the occurrence of cascades of topological changes, in response to a small stress increment above the yield stress [11].

The size of the cascades was found to increase with the liquid fraction [11], possibly tending to infinity, but it was not feasible to fully explore the wet limit, at which point the size was expected to diverge. In the present paper we return to this phenomenon, using a progressive increase of liquid fraction, rather than stress, as the small perturbation. In doing so, we also revive the PLAT program, which has scarcely been used in the intervening years, due to the problems of maintaining a functioning graphical interface in an ever changing software environment, and a tendency to stall in the wet limit. We do not use the Surface Evolver program [12] which is commonly used in foam physics, since the $2 \mathrm{D}$ version currently requires a finite contact angle which has a strong effect in the wet limit. The implications of this will be discussed in a future paper [13].

\section{Simulations of $2 \mathrm{D}$ foams using PLAT}

\subsection{The PLAT Software}

PLAT $[5,6,7,8]$, as the name suggests, is a software package for the simulation of a foam in 2D based on the direct implementation of Plateau's Laws, rather than an energy minimisation routine.

In a 2D foam, cell walls and liquid-gas interfaces are circular arcs which are constrained to meet smoothly at vertices, see Fig 1 . The radius of curvature of each arc, $r$, is determined by the Laplace law: $r=\gamma /\left(p_{i}-p_{b}\right)$ for liquid-gas interfaces and $r=2 \gamma /\left(p_{i}-p_{j}\right)$ for cell walls between bubbles. Here, $p_{i}$ and $p_{j}$ are the pressures in bubbles $i$ and $j$ and $p_{b}$ is the Plateau border pressure, set equal in all Plateau borders for a given value of liquid fraction $\phi$. Surface tension is denoted by $\gamma$.

The simulations are initialised as follows: samples are generated by filling a square box (see Fig. 2) with a random Delauney tessellation of triangles, which is inverted to obtain its dual graph - a Voronoi network. The Delauney tessellation is constructed by first randomly scattering $n$ points in the simulation box. The randomness of bubble areas is controlled by specifying a minimum separation between points, the so-called hard disk parameter as described by Weaire and Kermode [14]. We characterise the polydispersity of our samples by $\mu_{2}^{A}$, the variance of the cell areas, normalised by dividing by the square of the mean cell area. For the data presented here $\mu_{2}^{A}=0.02 \pm$ 0.004 .

The resulting Voronoi network is converted to a relatively dry foam, not yet equilibrated, by "decorating" its vertices with small three-sided Plateau borders at equal pressures. This initialisation procedure is provided by PLAT and is based on the pro- 


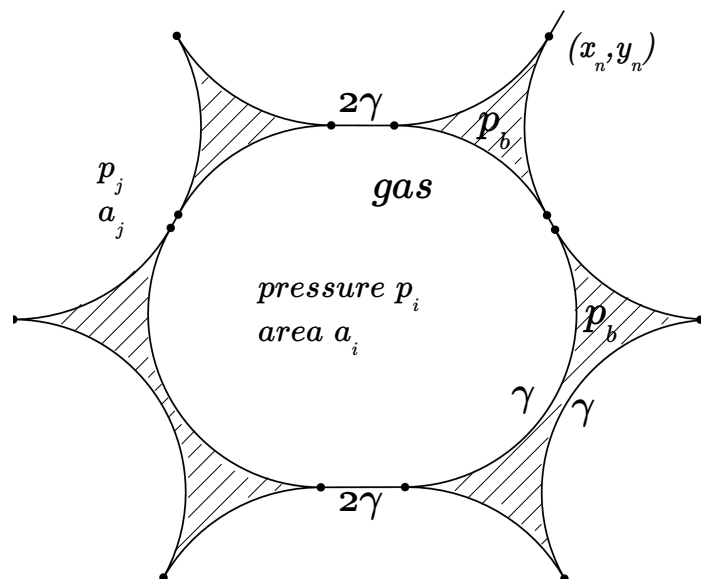

Figure 1. Schematic of a single bubble (or cell) in the PLAT representation of a foam. $\left(x_{n}, y_{n}\right)$ is the position of vertex $n, \gamma$ the interfacial surface tension, $p_{b}$ the Plateau border pressure, $p_{i}$ the gas pressure of bubble $i$, and $a_{i}$ the area of bubble $i$.

cedure described by Kermode and Weaire [15].

The decorated Voronoi network is equilibrated into a 2D foam by adjusting cell pressures and vertex positions in order to fulfil the constraints of fixed cell areas and smoothly meeting arcs. Convergence is determined from the magnitude of the maximum change in the vertex positions. For a more detailed discussion of the PLAT representation of a foam and the equilibration process see [6].

There are two types of topological changes implemented during equilibration. Cells loose contact when the vertices at either end of their shared cell-cell arc come within a minimum of each other, or pass each other. Cells come into contact when their corresponding cell-border arcs overlap across a Plateau border.

If a three-sided cell looses a contact, it does not turn into a 'rattler', but remains attached to its remaining neighbours. This procedure has been chosen because in this case the bubble remains part of the foam network and can be dealt with easily. Even towards the wet limit the fraction of one and two sided bubbles combined is less than $4 \%$.

The present simulation progresses by increasing the liquid fraction in steps of $\Delta \phi=$ 0.001 and equilibrating at each step. Increases in liquid fraction are performed by proportionally reducing bubble areas. This involves decreasing the cell pressures in order to reduce the curvature of the liquid-gas interfaces, followed by the equilibration of vertex positions. The pressure of every cell undergoes one iteration before each vertex position undergoes one iteration, and the two are converged together in the equilibration process. Calculations of foam energy or average coordination number are made after each equilibration.

Examples of structures simulated by PLAT are shown in Fig. 2. It is the natural extension to wet foam of the earlier 2D-FROTH simulation method of Weaire and Kermode $[16,14,15]$ for dry foam. Doubts were expressed at the time of the inception of FROTH as to whether the method might encounter anomalous equilibrium configurations not representative of a real $2 \mathrm{D}$ foam, but there is no evidence that this is the case in practice. However, PLAT exhibits a tendency to no longer succeed in equilibrating the foam structure at high liquid fractions for systems larger than about 20 bubbles. 

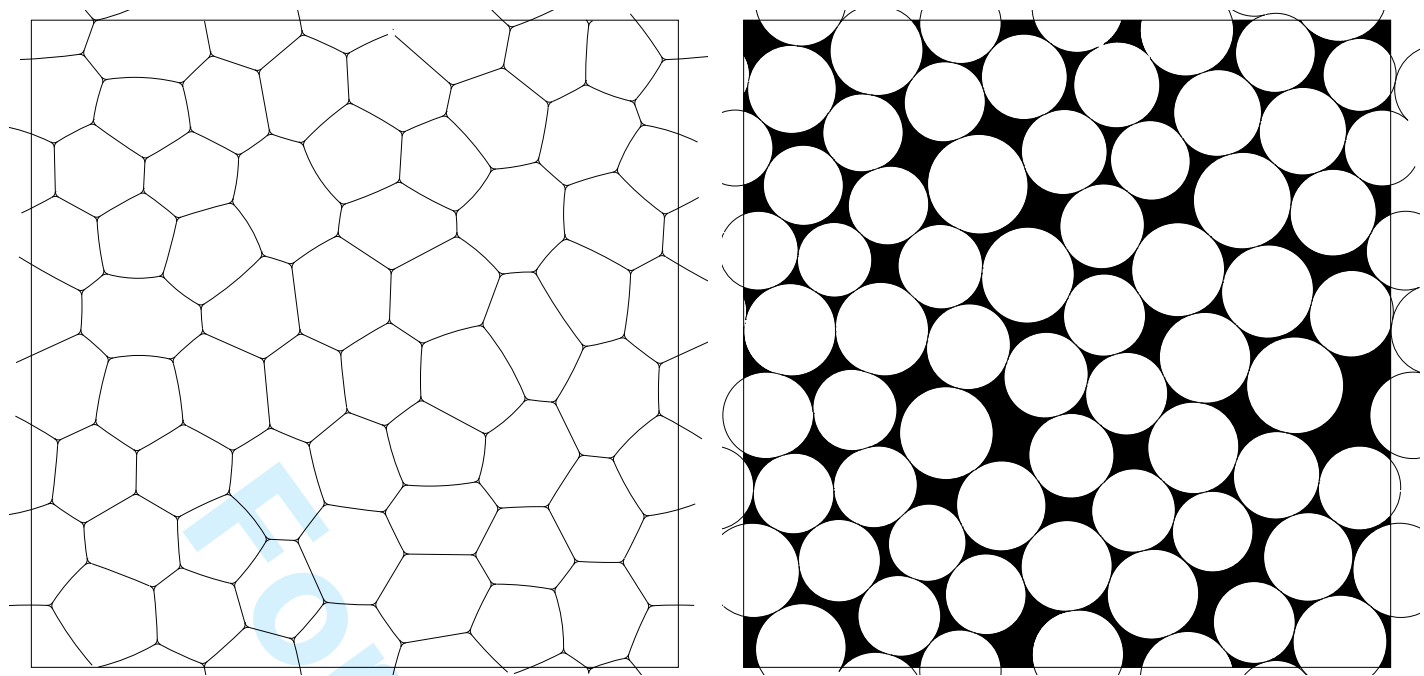

Figure 2. Example of initial (dry, $\phi=0.002$ ) and final (wet, $\phi=0.165$ ) stages of a sequence of PLAT simulations for a foam with 60 bubbles. Note the periodic boundary conditions.

As Plateau borders tend to become semicircular, it is increasingly difficult to fit an arc of the correct curvature between its end points and the system no longer converges. This is indeed the potential difficulty that was anticipated by Weaire and Kermode $[16,14]$. In very wet foams, large many-sided Plateau borders are much more likely to be susceptible to involve such anomalous arcs. We have not yet definitively identified them, or attempted a refinement of the program.

To reduce size effects we would prefer to use samples that are as large as possible. However, the frequent failure of PLAT made it impractical to simulate a full range of liquid fraction for samples exceeding about 100 bubbles. We thus decided that each simulation would consist of only 60 bubbles, and the properties of the simulations would be averaged over multiple simulations. For the purposes of this paper, all simulations are included in the averaged results, regardless of the liquid fraction reached. For details of averaging and statistics, see Appendix A.

Up to a liquid fraction of approximately 0.06, we have at least 500000 successful simulations, while after this point this number decreases roughly linearly towards zero at the wet limit $\phi=0.16$ (see A).

Before proceeding to the analysis of rearrangements we shall present results for various basic quantities of interest, complementing and completing previously published data from PLAT $[8,5,6,11,17]$.

\subsection{Variation of Energy with Liquid Fraction}

The energy $E(\phi)$ of a $2 \mathrm{D}$ foam with given bubble area distribution is simply the sum of the line length of all the bubbles in the foam times the (constant) line (surface in 3D) tension $\gamma$ (counting each side of a contact line separately). The wet limit energy $E_{0}$ of a 2 D foam with a contact angle of zero corresponds to the sum of the perimeters of circular disks having the same area distribution.

Fig. 3 shows the variation of the reduced excess energy $\varepsilon(\phi)$, defined as

$$
\varepsilon(\phi)=\frac{E(\phi)-E_{0}}{E_{0}}
$$




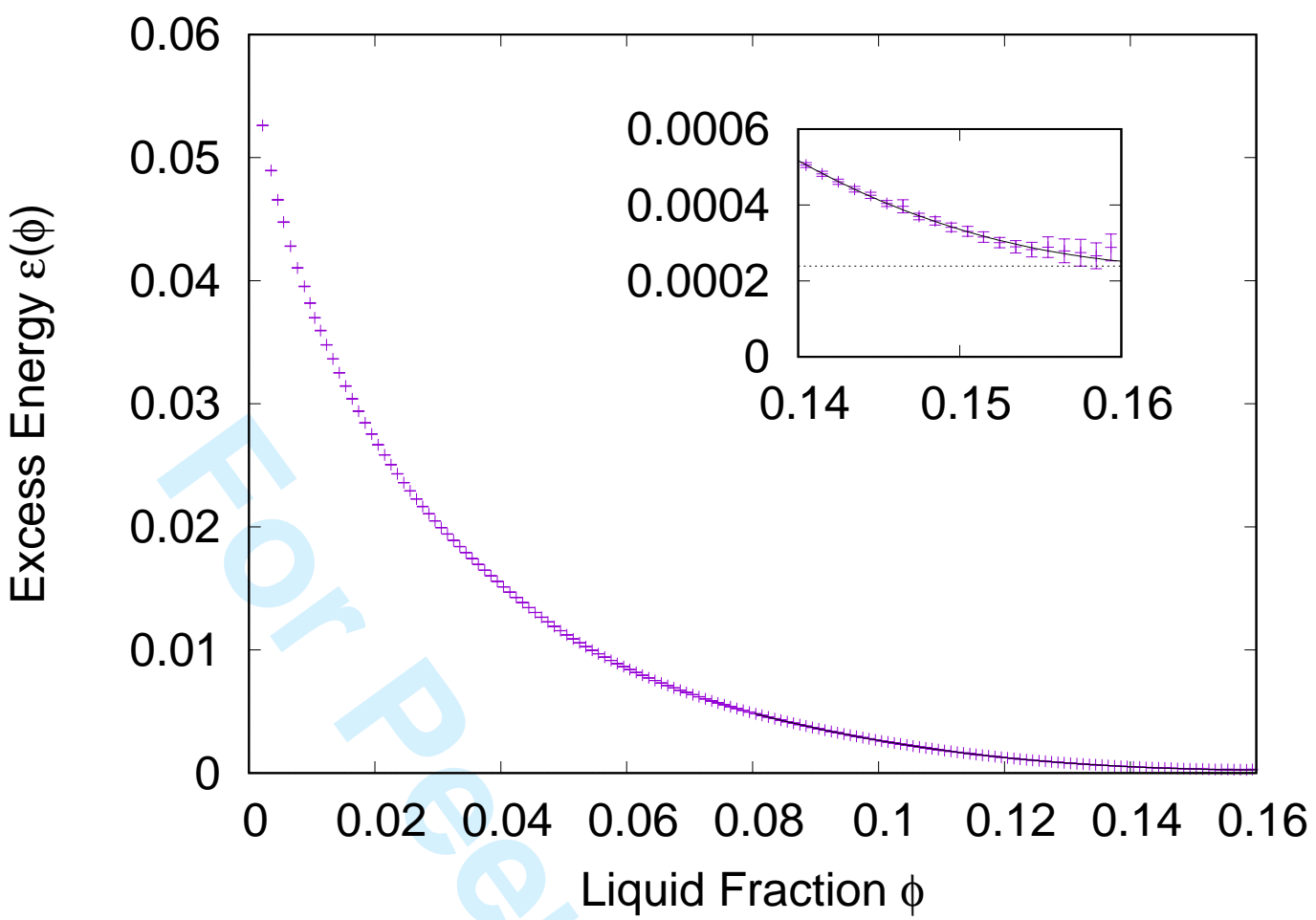

Figure 3. Variation of reduced excess energy $\varepsilon(\phi)$ with liquid fraction $\phi$. The function given by Eqn. 2 is shown as the solid line with a small added constant. Inset shows the wet limit.

as a function of liquid fraction. Close to the wet limit this is well fitted by a quadratic function and a cubic component, with a small added constant (see Eqn. 2). This gives a critical liquid fraction of $\phi_{c}=0.166 \pm 0.005$ for the wet limit.

$$
\varepsilon(\phi)=0.31\left(\phi_{c}-\phi\right)^{2}+3.7\left(\phi_{c}-\phi\right)^{3}+2 \times 10^{-4} .
$$

This value of $\phi_{c}$ is consistent with other numerical results for 2D foams $[8,18]$, and experimental $[19,20]$ and numerical $[9,21]$ results for random packings of disks. However, this is a 4 parameter fit. A more accurate fit involving only 2 parameters is obtained from considering the variation of average coordination number with $\phi$ (see Sec. 2.3.1). This results in $\phi_{c}=0.159 \pm 0.001$ [18] and it is this value that we will use when discussing large scale rearrangements in Sec. 3. The very small offset (the last term in Eqn. 2) is barely visible on the scale of the main plot of Fig. 3. Further calculation with tighter criteria for convergence have indicated that this small discrepancy is due to limited convergence. Given the extensive nature of these calculations, as described above, we have not repeated them in full to investigate this further.

\subsection{Coordination Numbers}

\subsubsection{Average Coordination Number}

A quantity of general interest for packing problems is the coordination number Z, i.e. the average number of contacts of a particle, or a bubble, in our case. For a foam, this is a function of liquid fraction (void fraction). It decreases from $Z=6$ for the dry limit 


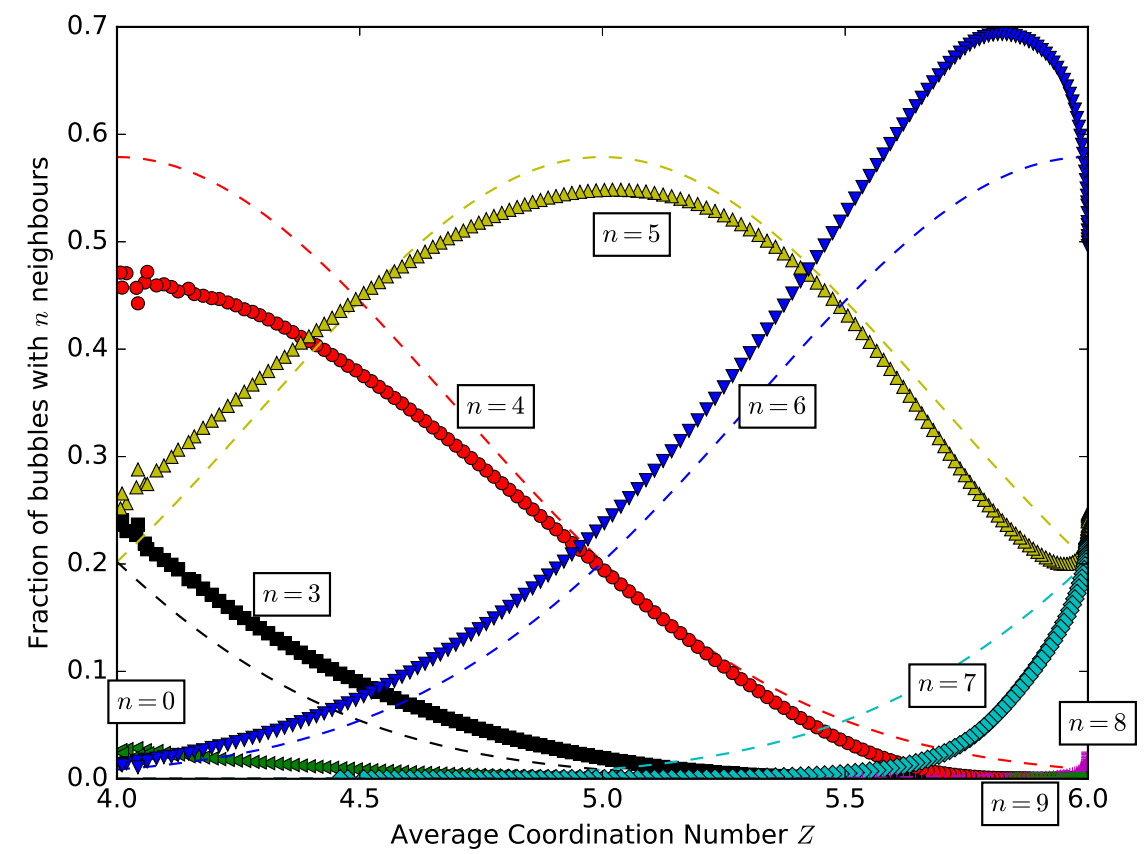

Figure 4. Simulation data for the fraction of $n$-sided bubbles as a function of average coordination number $Z$ (symbols) can be approximated by the functional form of Eqn.(3) (dashed lines), with only a single fit parameter $\sigma \simeq 0.684 \pm 0.004$ for all the data.

$(\phi=0)$ to $Z=4$ for the wet limit $\left(\phi=\phi_{c}\right)$ of an infinite system. The former value is essentially exact (from Euler's Theorem), the latter almost so (see [18] for details), with possible correction for occasional "rattlers" (i.e. bubbles that are free to move within the cage formed by their neighbours).

Surprisingly, $Z(\phi)$ varies linearly close to $\phi_{c}$, in contrast with the square root variation obtained from simulations and experiments of dense packings of soft disks [9]. A detailed discussion of this matter is presented in a further paper [18].

\subsubsection{Distribution of Coordination Number}

We now turn to the distrubution of coordination numbers and present an empirical procedure inspired in part by the analysis of van Hecke [22]. Fig. 4 shows distributions for the fraction of $n$-sided bubbles as a function of average coordination number $Z$. Here we propose the following function $f(n)$, involving only a single free parameter $\sigma$, as an adequate description of the data,

$$
f(n)=\frac{1}{\sigma \sqrt{2 \pi}} \exp \left(\frac{(Z-n)^{2}}{\sigma^{2}}\right)
$$

where $Z$ is the contact number, as above, and the value of $\sigma$ used in Fig. 4 is 0.684.

One notable feature of these statistics that is not represented in the simple fitting function is the steep variation of the curves as $Z$ tends to 6 . This may be understood as follows. It relates to variations close to the dry limit, where all of the Plateau borders are three-sided, "decorating" single vertices of the dry foam. As the liquid fraction $\phi$ is increased, rearrangements are progressively triggered as the borders come into contact, forming four-sided borders. In a nearly dry foam, these immediately trigger 


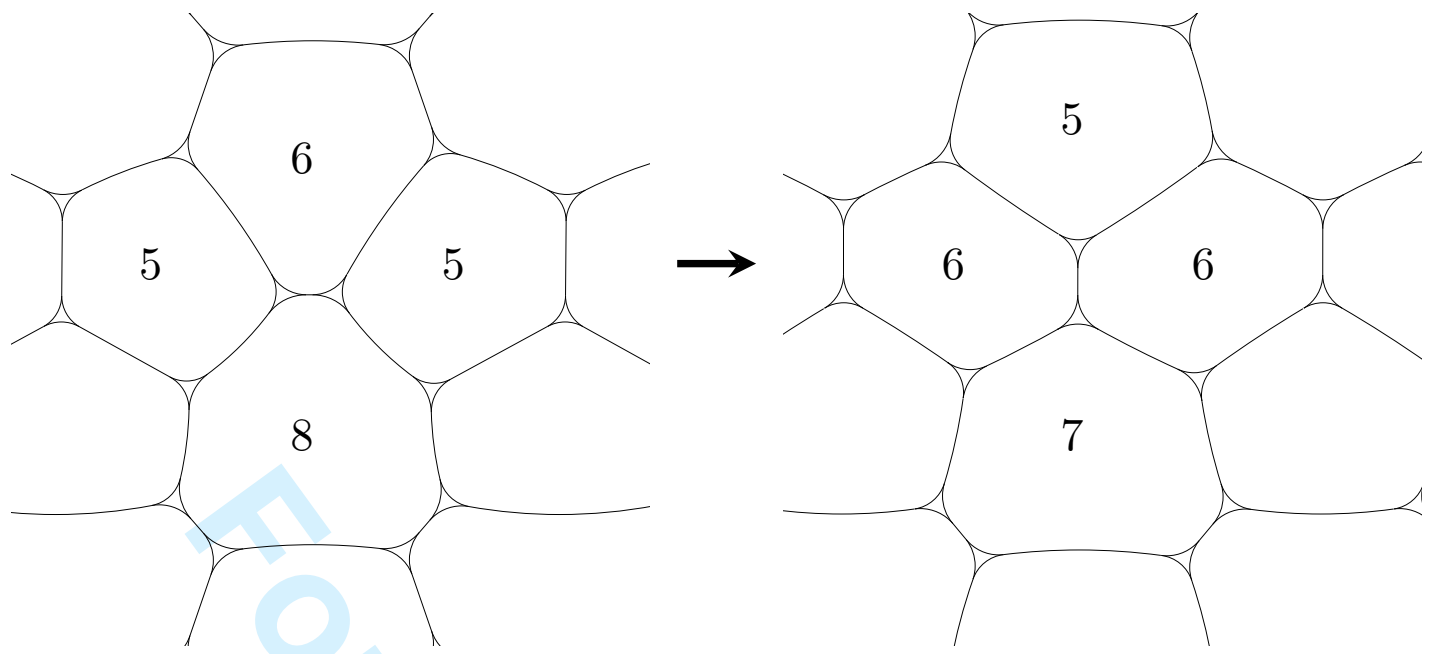

Figure 5. Example of a $\mathrm{T} 1$ transition where the mean contact number $Z$ is unchanged, but the distribution $f(n)$ does change. The numbers indicate the number of neighbours of each bubble.

further rearrangements to complete T1 changes [6]. Such combined changes have no effect on the mean contact number $Z$, which remains at the value 6 , but do affect the distribution $f(n)$, see Fig. 5. Accordingly, the derivatives $\frac{d f}{d Z}$ are infinite at the dry limit.

\section{Statistics of Bubble Rearrangements}

We have also performed a study of rearrangements, or topological changes, that take place as the liquid fraction is increased, especially as the wet limit is approached.

There are two types of elementary rearrangements in a 2D foam of finite liquid fraction, in which a contact between two bubbles is gained or lost - see Fig. 6. In what follows we will count the fraction of bubbles involved in rearrangements in any given step, rather than the rearrangements themselves. Another alternative would be to measure changes in the energy of the system, as did Durian for the more rudimentary bubble model $[9,23]$.

As the wet limit is approached it becomes particularly evident that more elementary rearrangements are provoked by a small increment of $\phi$.

The number of rearranged bubbles in one step is computed from the change of the adjacency matrix (which has unit elements $i j$ where bubbles $i$ and $j$ are in contact, zero otherwise) between two equilibrium states. Shown in Fig. 7 is the fraction of bubbles involved in rearrangements (as averaged over all our simulations) when increasing liquid fraction from $\phi$ to $\phi+\Delta \phi$ in small increments $\Delta \phi=0.001$. Since this average depends on the size of $\Delta \phi$ we have repeated the calculation for a range of values of $\Delta \phi$ and used this data to extrapolate to the limit $\Delta \phi \rightarrow 0$. The result of this is shown as dashed line in Fig. 7; see Appendix B for details.

We also generate histograms of the number of rearranged bubbles per step for all our simulation data at each liquid fraction. Viewing these histograms on a semi-log scale reveals that the tail of the distribution can be well approximated by an exponential distribution (see Fig. 8, top) of the form: 

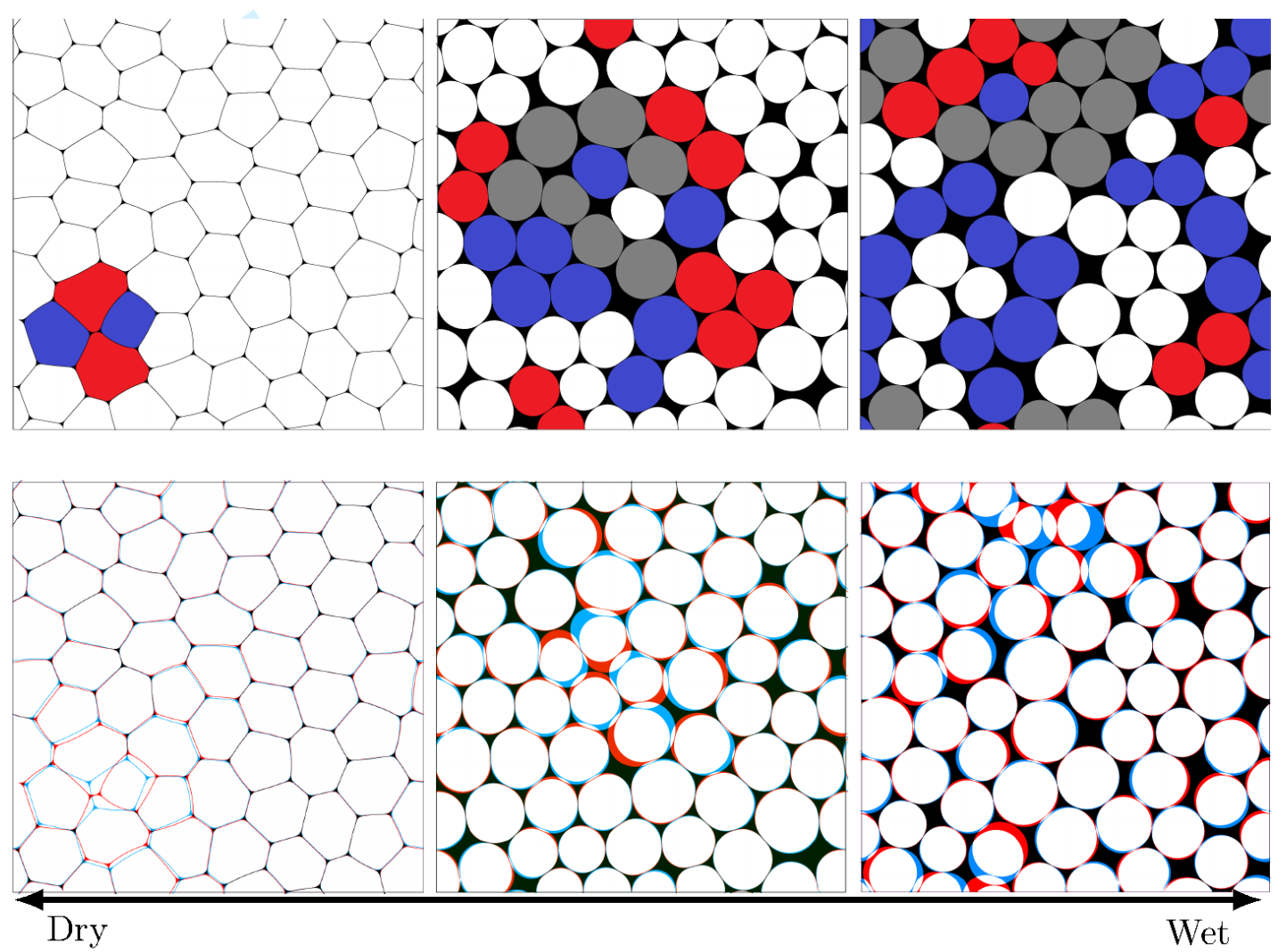

Figure 6. Two alternative illustrations of rearrangements due to a small increase in liquid fraction. Top row - Red bubbles lose contacts, blue bubbles gain contacts, and gray bubbles both gain and lose contacts. Bottom row - Red is the previous configuration, blue is the new configuration, and black is unchanged. Left: in a dry foam $(\phi=0.006)$, an increase in liquid fraction $\Delta \phi=0.001$ only leads to localised rearrangements. Centre: example of extensive rearrangements in a moderately wet foam $(\phi=0.112)$. Right: Rearrangements involving nearly $2 / 3$ of all bubbles in a foam $(\phi=0.149)$ close to the critical liquid fraction. Note that the regions in which changes occur appear spatially connected. 


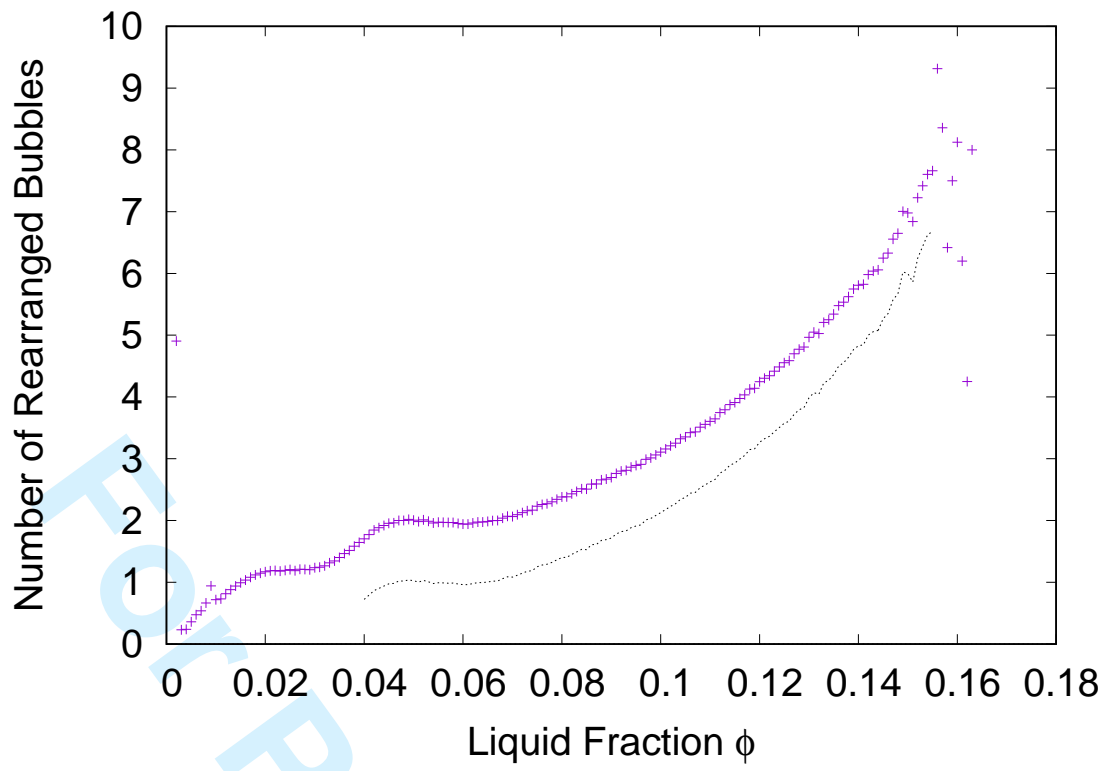

Figure 7. Fraction of rearranged bubbles in a sample due to an increase in liquid fraction by $\Delta \phi$ as a function of liquid fraction $\phi$. Data points are an average of $10^{5}$ simulations containing 60 bubbles each, with $\Delta \phi=0.001$. The dashed line corresponds to an extrapolation to the limit: $\Delta \phi \rightarrow 0$. See Appendix B.

$$
p(n)=\lambda \exp (-\lambda n)
$$

Here $\lambda$ is called the decay parameter, for an exponential distribution $1 / \lambda$ equals the mean of $n$. Instead of fitting 15 different exponentials (corresponding to the 15 datasets shown in Fig. 8 (top)), resulting in 15 values for $\lambda$ as a function of $\phi$ we have chosen to rescale both the $\mathrm{x}$ and $\mathrm{y}$ axis. This leads to a collapse of the data, as shown in Fig. 8 (centre). This rescaled dataset is fitted to Eqn. 4, resulting in a decay parameter of $\lambda_{0}=17 \pm 2$. To get $\lambda(\phi)$ we divide $\lambda_{0}$ by the square of the scale factor as a function of $\phi$. Fig. 8 (bottom), shows the variation of $1 / \lambda$ with liquid fraction $\phi$. In the wet limit the inverse decay parameter tends quadratically to a constant of $1 / \lambda\left(\phi_{c}\right)=0.150 \pm 0.001$, whose value or origin is as yet unclear.

Rather than describing our data with an exponential, we have also probed for a power law. However, the limited resolution of our data makes this difficult. Regardless, preliminary analysis indicates that the exponent would decrease in the limit $\phi \rightarrow \phi_{c}$, making us believe that the exponential distribution is a more appropriate model for the data.

There is a variety of physical systems which exhibit avalanches, ranging from earthquakes [24] and random fuse networks [25] to piles of rice [26]. However, their statistics are usually described by power-laws of the form $p(n) \sim n^{-\alpha}$, where the exponent $\alpha$ has a value between 1 and 2 . Indeed, power-laws are often seen as a signature feature of complex systems [27], which also include foams [28] and have been found, for example, in the statistics of particle rearrangements in flowing colloidal suspensions [29] and the statistics of popping bubbles in collapsing foams [30].

Power law distributions of changes in energy with an exponential cut-off for high energies have been observed in simulations by Durian [23] in packings of soft disks under shear. For this system it has been suggested that a pure power-law distribution 

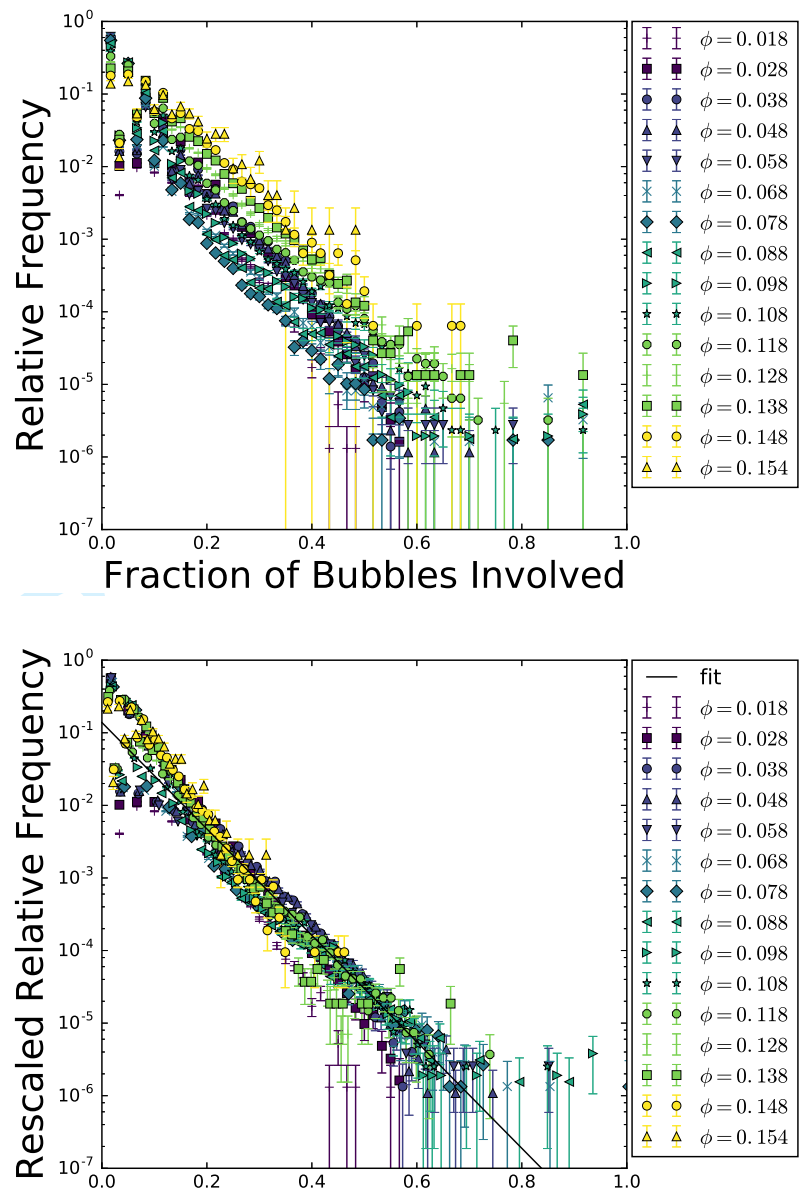

Rescaled Fraction of Bubbles Involved

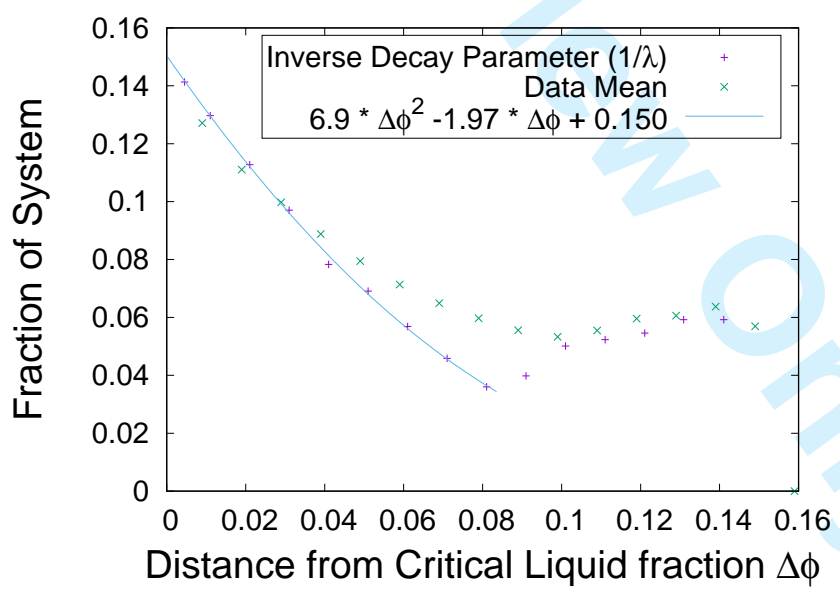

Figure 8. Top: Semi-log plot of histograms of the fraction of bubbles involved in rearrangements. Centre: Manually rescaled histograms. The y-axis has been multiplied by the same scale factor that the $\mathrm{x}$-axis was divided by to preserve the normalisation of the data. Solid line is a straight line fit with slope $\lambda_{0}=17 \pm 2$, showing that the tail is well approximated by an exponential form of Eqn. 4. Bottom: Variation of the decay parameter $\lambda$ with liquid fraction $\phi$. This is obtained by dividing $\lambda_{0}$ from (centre) by the square of the $\phi$ dependant scale factor. The solid line is a fit of the form $(6.9 \pm 0.9) \times\left(\phi_{c}-\phi\right)^{2}+(-1.97 \pm 0.07) \times\left(\phi_{c}-\phi\right)+$ $(0.150 \pm 0.001)$. Also shown is the mean of the fraction of bubbles involved in rearrangements as a function of liquid fraction (see text). 
only occurs at the wet limit [31]. Such simulations, where bubbles are modeled as overlapping disks, are successful in reproducing many rheological properties of foams, but recent work points to marked differences regarding the variation of average coordination number $Z(\phi)$ in the wet limit [18].

A transition between power law and exponentially distributed avalanches was observed by Ritacco et. al [32] and Frette et. al [26]. The former was for coalescence of bubbles in a bubble raft, while the latter was observed in sliding grains in rice piles. In both cases, the statistics were controlled by the dissipative forces in each system, viscosity and friction respectively. Our simulations are quasi-static and therefore involve no dynamic processes or dissipative forces. We are therefore unable to find an analogous control parameter for the distribution of our large scale rearrangements and the reason why the distribution of rearrangements in our simulations follows the exponential form of Eqn. 4 remains elusive at this stage. There have been experiments with 3D foams [33] for which avalanches of rearrangements following an increase in liquid fraction have been observed, but there is no statistical data available.

\section{Conclusions}

We have presented a detailed study of the statistics of 2D foams, spanning the entire range of liquid fraction from the dry to the wet limit. This was based on extensive computer simulations that were not practical when PLAT was developed in 1990. The reconstituted program still has difficulty in coping with the wet limit, which should be addressed in future. However, we have succeeded in calculating the excess energy of foam, the distribution of cell sides, and the number of rearrangements over the full range of liquid fraction.

An alternative approach to simulate wet foams with the Surface Evolver software [34] is associated with different complications. As it stands this software uses finite contact angles and we show in an upcoming paper [13] that largely suppresses the large scale rearrangements analysed here.

In addition to providing further possibilities for analysing properties of $2 \mathrm{D}$ foams, we hope that our numerical results stimulate new experiments. These may be realised in a variety of ways e.g., by placing a monolayer of foam between two glass plates (for an elegant recent setup see Durian [35]), or by covering a Bragg raft with a glass plate. Although a 2D liquid fraction is not well defined in any of these cases, such experiments should give an indication whether large scale rearrangements occur on small increases of liquid fraction in the wet limit, and increase in the manner suggested here.

\section{References}

[1] D. Weaire and S. Hutzler. The Physics of Foams. Oxford University Press, Oxford, 1999.

[2] I. Cantat, S. Cohen-Addad, F. Elias, F. Graner, R. Höhler, O. Pitois, F. Rouyer, and A. Saint-Jalmes. Les mousses - Structure et dynamique. Éditions Belin, Paris, 2010.

[3] Wiebke Drenckhan and Stefan Hutzler. Structure and energy of liquid foams. Advances in Colloid and Interface Science, 224:1 - 16, 2015.

[4] HM Princen and AD Kiss. Rheology of foams and highly concentrated emulsions: Iii. static shear modulus. Journal of Colloid and Interface Science, 112(2):427-437, 
1986.

[5] F. Bolton and D. Weaire. The effects of Plateau borders in the two-dimensional soap froth. I. Decoration lemma and diffusion theorem. Phil. Mag. B, 63:795-809, 1991.

[6] F. Bolton and D. Weaire. The effects of Plateau borders in the two-dimensional soap froth. II. General simulation and analysis of rigidity loss transition. Phil. Mag. B, 65:473-487, 1992.

[7] F. Bolton. Software PLAT: A computer code for simulating two-dimensional liquid foams. https://github.com/fbolton/plat, 1996.

[8] F. Bolton and D. Weaire. Rigidity loss transition in a disordered 2D froth. Physical Review Letters, 65:3449, 1990.

[9] D. J. Durian. Foam mechanics at the bubble scale. Phys. Rev. Lett., 75:4780-4783, 1995.

[10] H. M. Princen. Rheology of Foams and Highly Concentrated Emulsions II. Experimental Study of the Yield Stress and Wall Effects for Concentrated Oil-in-Water Emulsions. J. Colloid Interface Sci., 105:150-171, 1985.

[11] S. Hutzler, D. Weaire, and F. Bolton. The effects of Plateau borders in the twodimensional soap froth, III. Further results. Phil. Mag. B, 71:277, 1995.

[12] K. Brakke. http://www.susqu.edu/brakke/evolver/evolver.html.

[13] D. Weaire, S. Cox, and S. Hutzler. Finite Contact Angles in Wet 2D Foams. In preparation, 2016.

[14] D. Weaire and J. P. Kermode. Computer simulation of a two-dimensional soap froth. II. Analysis of results. Phil. Mag. B, 50:379-395, 1984.

[15] J. P. Kermode and D. Weaire. 2d-froth: A programme for the investigation of 2-dimensional froths. Comp. Phys. Commun, 60:75-109, 1990.

[16] D. Weaire and J. P. Kermode. Computer simulation of a two-dimensional soap froth. I. Method and motivation. Phil. Mag. B, 48:245-259, 1983.

[17] S Hutzler and D Weaire. The osmotic pressure of a two-dimensional disordered foam. Journal of Physics: Condensed Matter, 7(47):L657, 1995.

[18] Winkelmann, J. and Dunne, F.F. and Langlois, V. J. and Möbius M. E. and Weaire, D. and Hutzler, S. 2d foams above the jamming transition: Deformation matters. In preparation, 2016.

[19] D Bideau and JP Troadec. Compacity and mean coordination number of dense packings of hard discs. Journal of Physics C: Solid State Physics, 17(28):L731, 1984.

[20] T. S. Majmudar, M. Sperl, S. Luding, and R. P. Behringer. Jamming transition in granular systems. Phys. Rev. Lett., 98:058001, Jan 2007.

[21] Qicheng Sun and Stefan Hutzler. Lattice gas simulations of two-dimensional liquid foams. Rheologica Acta, 43(5):567-574, 2004.

[22] G. Katgert and M. van Hecke. Jamming and geometry of two-dimensional foams. EPL (Europhysics Letters), 92(3):34002, 2010.

[23] D. J. Durian. Bubble-scale model of foam mechanics: Melting, nonlinear behavior, and avalanches. Phys. Rev. E, 55:1739-1751, 1997.

[24] Beno Gutenberg and Charles Francis Richter. Magnitude and energy of earthquakes. Science, 83(2147):183-185, 1936.

[25] L De Arcangelis and HJ Herrmann. Scaling and multiscaling laws in random fuse networks. Physical Review B, 39(4):2678, 1989.

[26] Vidar Frette, Kim Christensen, Anders Malthe-Sørenssen, Jens Feder, Torstein Jøssang, and Paul Meakin. Avalanche dynamics in a pile of rice. Nature, 379(6560):49-52, 1996. 


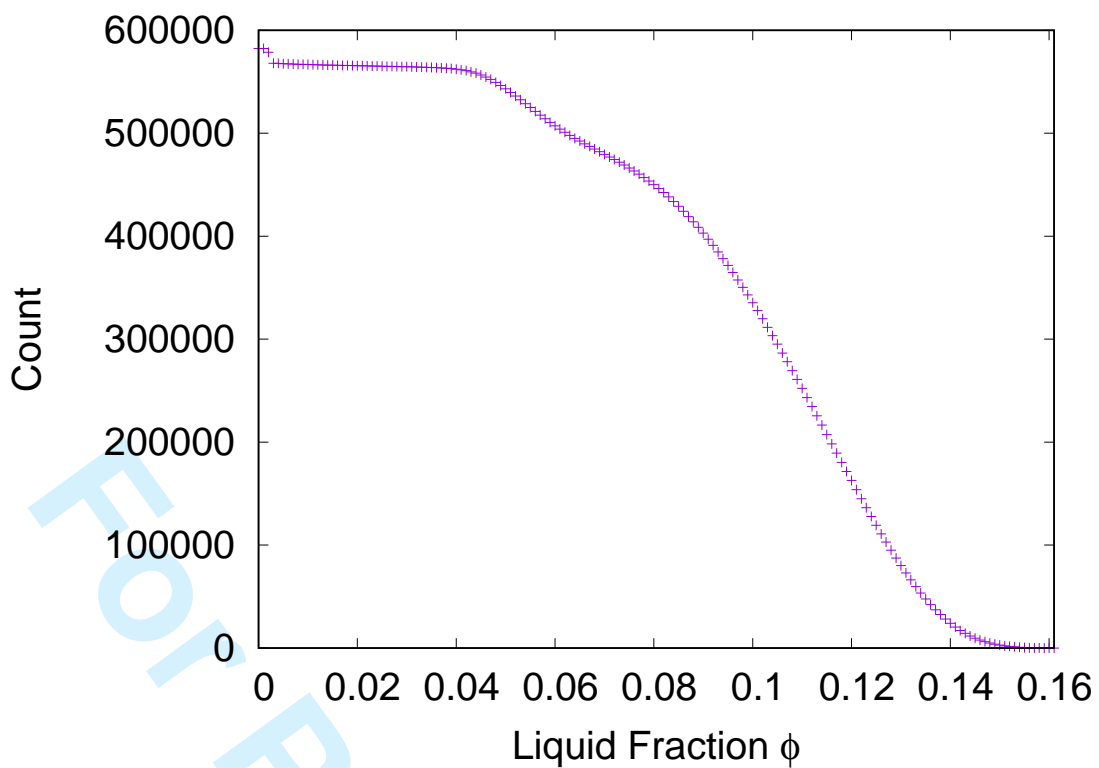

Figure A1. Number of successful simulations as a function of liquid fraction. The decrease in successful simulations at higher liquid fractions is due to stalls of the PLAT software (see Sec. 2.1).

[27] Péter Érdi. Complexity Explained. Springer-Verlag, Berlin Heidelberg, 2008.

[28] D. Weaire and S. Hutzler. Foam as a complex system. J. Phys.: Condens. Matter, 21:4227, 2009.

[29] K. N. Nordstrom, J. P. Gollub, and D. J. Durian. Dynamical heterogeneity in soft-particle suspensions under shear. Phys. Rev. E, 84:021403, Aug 2011.

[30] N. Vandewalle, J. F. Lentz, S. Dorbolo, and F. Brisbois. Avalanches of popping bubbles in collapsing foams. Physical Review Letters, 86:179-182, 2001.

[31] Shubha Tewari, Dylan Schiemann, Douglas J. Durian, Charles M. Knobler, Stephen A. Langer, and Andrea J. Liu. Statistics of shear-induced rearrangements in a two-dimensional model foam. Phys. Rev. E, 60:4385-4396, Oct 1999.

[32] H. Ritacco, F. Kiefer, and D. Langevin. Lifetime of bubble rafts: Cooperativity and avalanches. Physical Review Letters, 98:244501, 2007.

[33] S. Hutzler, D. Weaire, and S. Shah. Bubble sorting in a foam under forced drainage. Phil. Mag. Lett., 80:41-48, 2000.

[34] K. A. Brakke. The surface evolver. Experimental Mathematics, 1:141-165, 1992.

[35] A. E. Roth, C. D. Jones, and D. J. Durian. Bubble statistics and coarsening dynamics for quasi-two-dimensional foams with increasing liquid content. Phys. Rev. E, 87:042304, Apr 2013.

\section{Appendix A. Averaging of simulations and statistics}

All the results presented in this paper are calculated and averaged across almost 600000 simulations. Not all simulation ran to completion, this can be seen in the decline of the statistics shown in Fig. A1. These statistics nevertheless appear to be more than adequate for our purposes.

Such a large number of simulations would have been entirely unachievable in the 1990s when PLAT was conceived; the (successful) calculation of one single 60-cell sample for a range of values of liquid fraction would have taken several hours. 


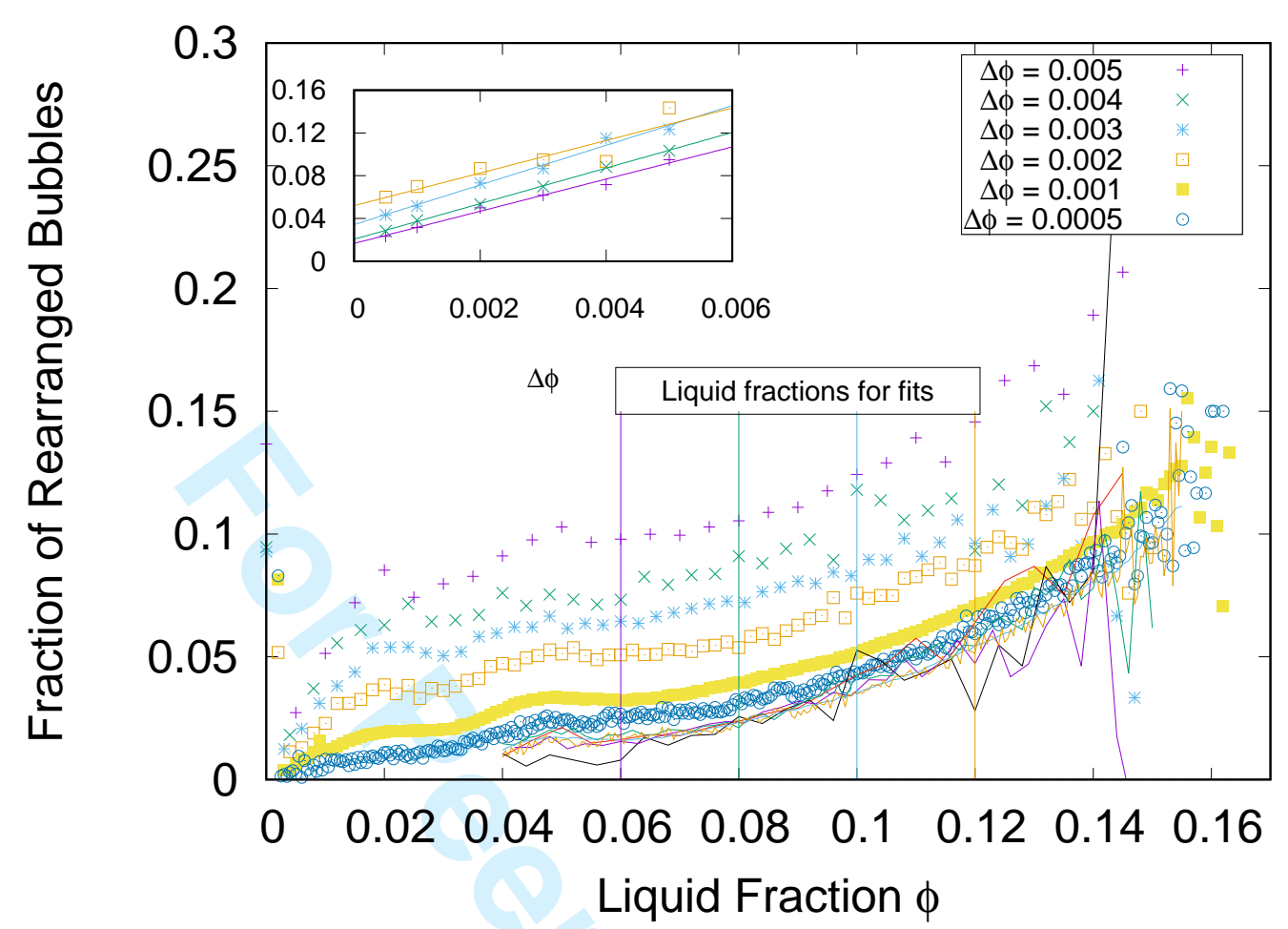

Figure B1. Fraction of rearranged bubbles due to an increase in liquid fraction by $\Delta \phi$ as a function of liquid fraction $\phi$ (data averaged over $10^{5}$ simulations for $(\Delta \phi=0.001)$ and $10^{3}$ simulations for all other values of $\Delta \phi)$. Inset: Fraction of rearrangements as a function of stepsize $\Delta \phi$. Data sampled at liquid fractions indicated on the main figure. Solid lines mark the interpolations as $\Delta \phi \rightarrow 0$.

\section{Appendix B. Extrapolation of $\Delta \phi \rightarrow 0$}

The main plot of Fig B1 shows the average fraction of rearranged bubbles due to an increase in liquid fraction from $\phi$ to $\phi+\Delta \phi$ for six different values of $\Delta \phi$. The dependence of this average fraction on $\Delta \phi$ can be studied by taking vertical slices through this data. We have done this for four different values of $\phi$, indicated by the lines labeled 'Liquid fractions for fits'. The result is shown in the inset plot. The average fraction of rearranged bubbles is seen to decreases to a non-zero value when we extrapolate $\Delta \phi$ to zero. The straight line fits in the inset all have very similar slopes, indicating that the linear shift due to non-zero $\Delta \phi$ is constant, so we take an average of the fits, giving an offset of $-(16.4 \pm 0.5) \Delta \phi$. 